\author{
Moorkath Nandakumaran ${ }^{\mathrm{a}}$ \\ Ma'asoumah Makhseed ${ }^{\mathrm{a}}$ \\ Samer Al-Rayyes ${ }^{\mathrm{a}}$ \\ Thattaruparambil N. \\ Sugathan ${ }^{\mathrm{b}}$ \\ a Departments of Obstetrics and \\ Gynecology, and \\ b Community Medicine, Faculty of \\ Medicine, Kuwait University, \\ Kuwait
}

\section{Transport Kinetics of Alpha-Aminoisobutyric Acid in the Perfused Human Placental Lobule in vitro}

\begin{abstract}
Objective: The transports kinetics of $\alpha$-aminoisobutyric acid (AIB) has been investigated in the human placenta in vitro using perfusion of isolated placental lobules. Methods: NCTC 135 tissue culture medium diluted with Earle's salt solution was used as the perfusate, and tritiated water was used as the reference marker. Results: The transport fraction of AIB averaged $13.2 \%$ of that of tritiated water in 5 single-bolus injection experiments. The differential transport rates for 10 , $25,50,75$ and $90 \%$ cumulative efflux in the fetal vein of the study and the reference substances differed significantly as well. The transport kinetic parameters as area under the curve, clearance, elimination constant, time for maximum response, absorption rate and elimination rate of the amino acid averaged $0.12,19.8,0.78,1.28,0.10$ and 0.06 times, respectively. Plotting of relative AIB transport ratios as a function of perfusion time demonstrated the active nature of amino acid transport in our perfusion conditions. Conclusions: The transport and kinetic parameters employed in the study may be useful in assessing dynamic placental membrane function of A type amino acids and other substances in varying experimental conditions as well as in disease states.
\end{abstract} (.................

\section{Introduction}

$\alpha$-Aminoisobutyric acid (AIB) has been widely used as a marker for placental permeation studies, both in humans [1,2] as well as in animals [3, 4]. Its nonmetabolizable nature has permitted the investigators to specifically explore amino acid transport independent of maternal and fetal metabolic influences. Transport studies of this amino acid in humans have centered mainly on in vitro perfusion of isolated placental lobules, focusing on

\begin{tabular}{ll}
\hline KARGER & ( 1998 S. Karger AG, Basel \\
1011-7571/98/0074-0264\$15.00/0 \\
$\begin{array}{l}\text { Fax +4161306 1234 } \\
\begin{array}{l}\text { E-Mail karger@karger.ch } \\
\text { www.karger.com }\end{array}\end{array}$ & $\begin{array}{l}\text { Accessible online at: } \\
\text { http://BioMedNet.com/karger }\end{array}$
\end{tabular}

Dr. M. Nandakumaran, Assistant Professor

Department of Obstetrics and Gynecology

Faculty of Medicine

Kuwait University, PO Box 24923

Safat 13110 (Kuwait) 
the steady state rather than the dynamic state of membrane function. Hence, we thought it interesting to explore the transport kinetics of AIB in the perfused placental tissue, using a modified experimental protocol and using specifically designed transport parameters. The bolus injection method was chosen when compared to the steady-state perfusion method, since the former was found to be more suitable than the latter to assess dynamic transport function characteristics of the substances in question. Previous studies in our laboratory $[5,6]$ had established that such a protocol served to delineate transport kinetics of study substances independent of minor variations in factors such as perfusion flow rate, dosage or membrane surface area. Tritiated water was used as the reference because of its flow-limited characteristics. Further, its use has permitted us to minimize possible experimental artifacts as physiological shunts or changes in the membrane surface area and to obtain control data, permitting comparison with similar studies in varying experimental or disease situations.

\section{Materials and Methods}

Human placentae were collected post partum after uncomplicated pregnancies, and perfusion of suitable placental lobules was performed as previously described [7-10]. Briefly, fetal vessels of an isolated placental lobule were cannulated and perfused with NCTC 135 (Sigma Chem. Co., St. Louis, Mo., USA) tissue culture medium - Earle's solution mixture [2] being the perfusate. Bovine serum albumin at a concentration of $1 \mathrm{~g} / \mathrm{l}$ was added to the perfusate to compensate for the absence of plasma proteins in the perfusion medium. Perfusion was effected using a Harvard digital pump. Flow rates of the perfusate in the fetal and maternal circuits were monitored by flow meters (Brooks R $215 \mathrm{~A}$ ), and perfusion pressure was monitored by mercury manometers. The viability of the preparation was assessed by determining the oxygen and glucose consumption and the $\mathrm{CO}_{2}$ production of the tissue during perfusion.

\section{Experimental Design}

After an initial washout period of $10 \mathrm{~min}, 1 \mu \mathrm{Ci}$ of tritiated water (specific activity: $5 \mathrm{mCi} / \mathrm{mmol}$, Amersham, UK) and $0.5 \mu \mathrm{Ci}$ of ${ }^{14} \mathrm{C}$-AIB (specific activity: $60 \mathrm{mCi} / \mathrm{mmol}$, Amersham) were injected as $100-\mu \mathrm{l}$ bolus into the maternal circulation at a site close to the insertion of the microcannulas in the basal plate. After a period of $1 \mathrm{~min}$, serial perfusate samples were collected from the fetal venous outflow every $30 \mathrm{~s}$ for a period of $5 \mathrm{~min}$. The time lag of $1 \mathrm{~min}$ before the start of the sample collection was determined in previous control experiments, by injecting a $100-\mu 1$ bolus of Indian ink into the maternal circulation at the preselected site and by noting the time interval for the dye to first appear on the basal plate. The effective study period of $5 \mathrm{~min}$ was fixed after noting the time required for $90 \%$ of the injected radioactivity to appear in the combined fetal and maternal venous effluents in the control experiments, after taking into account the 1-min lag period mentioned earlier.

Radioactivity (dpm) in the different perfusate samples and injected boluses was determined by liquid scintillation counting (LKB Wallac model). Necessary precautions were taken to minimize errors from quenching and isotope crossover in counts, by adjusting the tritium and ${ }^{14} \mathrm{C}$ windows and by using standards with known radioactivity concentrations.

\section{Transport Parameters}

(1) The maternal-fetal transport of AIB and tritiated water was expressed as differential transport rate (TR), expressed as time in minutes for a given fraction to be transported across to the fetal vein [5].

\section{Efflux fraction $=$}

${ }^{3} \mathrm{H}$ or ${ }^{14} \mathrm{C}$ dpm in fetal venous sample total ${ }^{3} \mathrm{H}$ or ${ }^{14} \mathrm{C} \mathrm{dpm}$ in fetal vein for a period of $5 \mathrm{~min}$.

From the curve obtained by plotting various cumulative efflux fractions as a function of perfusion time, the time in minutes for an efflux of 10,25, 50, 75 and $90 \%$ of total venous efflux was then computed. Control studies had established that these efflux times, 6 for the study substances, varied little with increasing or decreasing doses for a given set of fetomaternal flow rates.

(2) The transport rate index (TI) of the test substance was then computed for $50 \%$ of the efflux by the following formula:

$\mathrm{TI}(\mathrm{AIB})=\frac{\mathrm{AIB} \mathrm{TR}}{\mathrm{H}_{2} \mathrm{O} \mathrm{TR}}$.

Med Principles Pract 1998;7:264-270 265 
(3) The transport fraction (TF) of the studied substances was calculated thus:

$\mathrm{TF}=$

$\underline{\text { total }{ }^{3} \mathrm{H} \text { or }{ }^{14} \mathrm{C} \mathrm{dpm} \text { in fetal venous efflux for a period of } 5 \mathrm{~min}}$ total ${ }^{3} \mathrm{H}$ or ${ }^{14} \mathrm{C}$ dpm in the injected bolus

Calculation of the ratio relating to the TF of the test studied substance to that of the reference substance will permit assessment of the TI of the former, independent of minor variations in flow rate, shunts or changes in membrane surface area.

(4) The area under the curve (AUC) of the studied substances was determined by trapezoid rule, assuming a two-compartment model and using the following formula $[5,6,11]$ :

$A U C=\sum_{I=1}^{n} \frac{[C(i+1)+C(i)] \times[t(i+1)-t(i)]}{2}+\frac{C(n)}{K_{e l}} ;$

where $\mathrm{C}=$ concentration of the substance in the sample, $\mathrm{t}=$ time in seconds of sample collection, $\mathrm{n}=$ number of experimental points, $\mathrm{C}_{\mathrm{n}}=$ last experimental point or measurement and $\mathrm{K}_{\mathrm{el}}=$ elimination constant .

Other kinetic parameters as clearance, $\mathrm{K}_{\mathrm{el}}, \mathrm{T}_{\max }$ (time for maximal response), absorption rate and elimination rate were determined using a computer program based on IMSL Fortran Subroutine software, specially adapted for statistical applications. The software was run on a VAX/VMS 6510 computer.

(5) To assess the active or passive nature of AIB transport, another transport parameter was also employed. The ratio of AIB label counts to tritiated water label counts was computed in the different perfusate samples in the fetal vein, and the ratios were plotted as a function of perfusion time for the whole duration of the experiment. It was premised that the ratio of the two labels in the injectate being constant, any increase in the relative AIB label ratio in the fetal venous effluent as a function of perfusion time would be indicative of the active nature of transport of the amino acid.

\section{Results}

Figure 1 shows the efflux pattern of tritiated water and AIB in a typical perfusion experiment. This pattern was observed in all 5 successful perfusions. Table 1 summarizes the differential transport rates of tritiated water and AIB for 10, 25, 50, 75 and 90\% efflux in the fetal vein. The AIB TR averaged $0.36 \pm$ $0.14,0.77 \pm 0.11,1.44 \pm 0.12,2.81 \pm 0.14$ and $3.52 \pm 0.17 \mathrm{~min}$, respectively, for 10,25 , 50,75 and $90 \%$ efflux fractions in the 5 perfusions, representing $1.61 \pm 0.08,1.59 \pm 0.11$, $1.84 \pm 0.09,1.62 \pm 0.18$ and $1.14 \pm 0.16$ times those of tritiated water values. The paired Student's $t$ test showed that the TR of both substances differed significantly $(\mathrm{p}<$ 0.05 ) for all the 5 efflux points investigated. The TI of AIB expressed as $\left(\mathrm{TR}_{50} \mathrm{AIB}\right) /\left(\mathrm{TR}_{50}\right.$ $\mathrm{H}_{2} \mathrm{O}$ ) ratio, averaged $1.84 \pm 0.09$ in the above experiments.

The fetal perfusate flow rate averaged 4.4 $\pm 0.3 \mathrm{ml} / \mathrm{min}$ while the maternal flow rate averaged $10.6 \pm 0.4 \mathrm{ml} / \mathrm{min}$ in the 4 successful perfusions. The cotyledon weight averaged $28.6 \pm 2.4 \mathrm{~g}$.

The TF of AIB averaged $0.22 \pm 0.08 \%$ of the injected amino acid dose in the 5 perfusions, while that of tritiated water averaged $1.48 \pm 0.07 \%$. Student's t test showed that the TF of AIB differed significantly from that of water $(p<0.05)$. The TF values of AIB represented $13.2 \pm 1.8 \%$ of the tritiated water $\mathrm{TF}$ in the 5 experiments.

Various transport kinetic parameters of study and reference substances in the 5 perfusions are shown in table 2 . When the 2 groups of data were subjected to the paired Student's $t$ test, AUC, clearance, $T_{\max }$, absorption rate and elimination rate were shown to be significantly different $(\mathrm{p}<0.05)$, while no significance could be shown $(p>0.05)$ between the 2 groups in the case of $\mathrm{K}_{\mathrm{el}}$, because of the interexperimental variability. AIB kinetic parameters such as AUC, clearance, $\mathrm{K}_{\mathrm{el}}, \mathrm{T}_{\max }$, absorption rate and elimination rate values averaged 56,185 $\pm 2,626 \mathrm{dpm}, 45.3 \pm 13.8 \mathrm{ml} /$ $\min , 3.06 \pm 0.84,124.19 \pm 6.9 \mathrm{~s}, 87 \pm 29$ $\mathrm{dpm}$ and $172 \pm 56 \mathrm{dpm}$, respectively, representing $0.12 \pm 0.02,19.8 \pm 2.2,0.78 \pm 0.14$, $1.28 \pm 0.06,0.10 \pm 0.02$ and $0.06 \pm 0.01$ times those of the corresponding tritiated wa- 


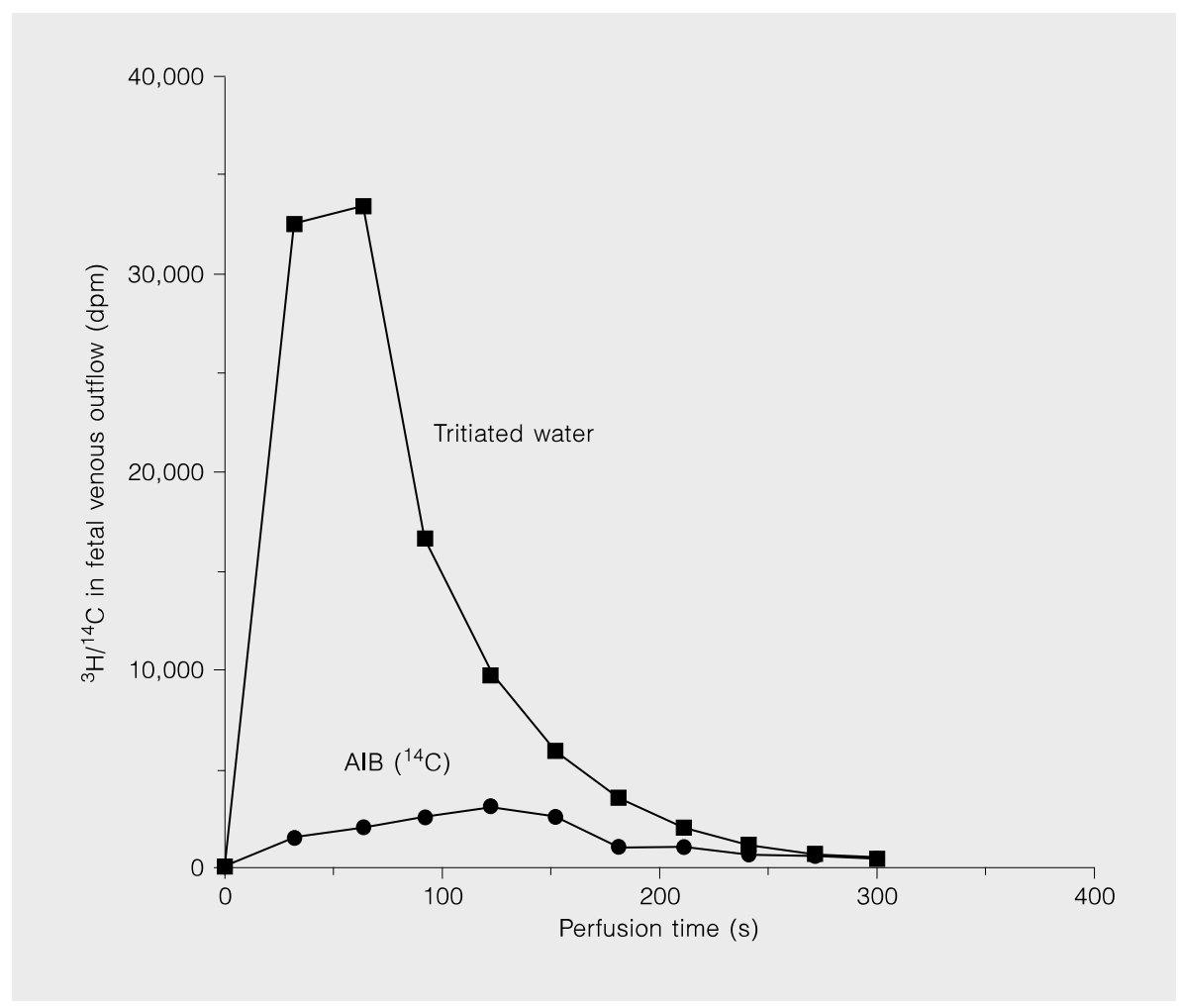

Fig. 1. Efflux pattern in the fetal vein of ${ }^{14} \mathrm{C}-\mathrm{AIB}$ and tritiated water in a typical perfusion experiment.

Table 1. Differential TR of AIB and tritiated water in normal placentae

\begin{tabular}{llllll}
\hline Substance & \multicolumn{5}{l}{ Differential TR, min } \\
\cline { 2 - 6 } & $\mathrm{TR}_{10}$ & $\mathrm{TR}_{25}$ & $\mathrm{TR}_{50}$ & $\mathrm{TR}_{75}$ & $\mathrm{TR}_{90}$ \\
\hline Tritiated water & $0.21 \pm 0.07$ & $0.46 \pm 0.12$ & $0.67 \pm 0.14$ & $1.64 \pm 0.15$ & $2.84 \pm 0.17$ \\
AIB & $0.36 \pm 0.14$ & $0.77 \pm 0.11$ & $1.44 \pm 0.12$ & $2.81 \pm 0.14$ & $3.52 \pm 0.17$
\end{tabular}

Values represent means + SEM of 5 successful perfusions.

ter values. Relative AIB TR, plotted as a function of perfusion time, is shown in figure 2. A strong correlation was shown between the 2 groups $\left(\mathrm{r}^{2}=0.972\right)$ when the data were subjected to linear-regression analysis by the least-square method.

Placental Transport Kinetics of a-Aminoisobutyric Acid

\section{Discussion}

The results on tritiated water transport kinetics are consistent with earlier reports $[5,6]$ of our research group. The data also fit well with those of international research groups

Med Principles Pract 1998;7:264-270 
Fig. 2. Relative TR of AIB in different perfusate samples, expressed in relation to corresponding tritiated water label counts. The results are expressed as means of 5 successful perfusions. The linear regression line was drawn using the equation: $\mathrm{y}=$ $3.6365^{-3}+3.8884^{-4 x} \cdot r^{2}=0.972$.

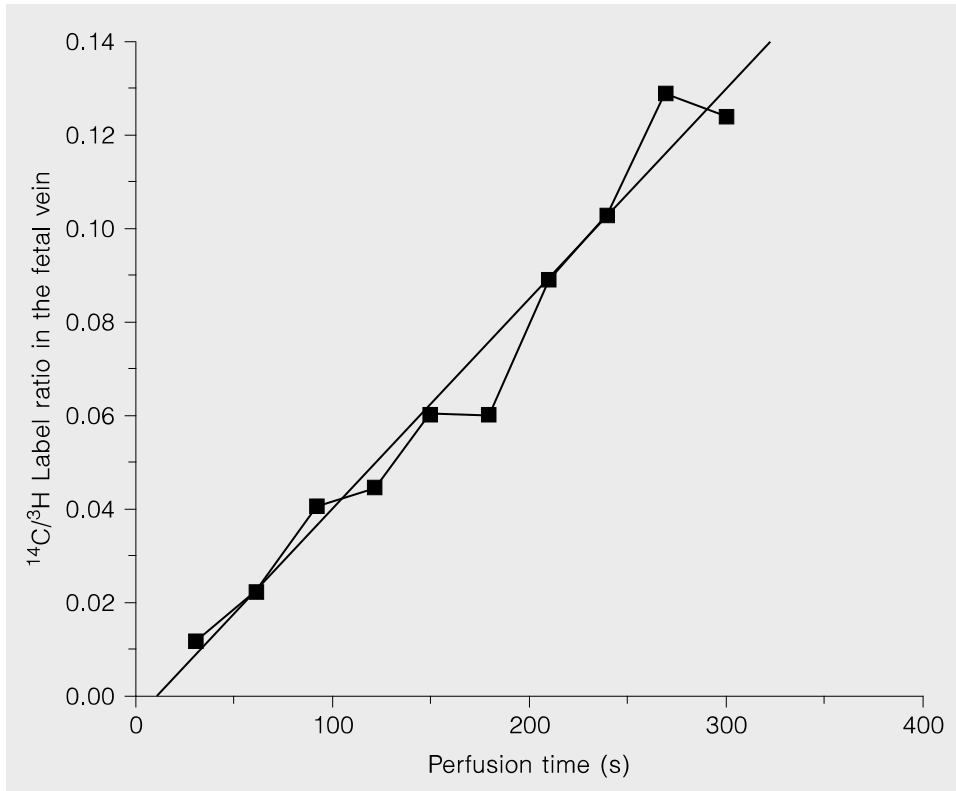

Table 2. Kinetic parameters of AIB and tritiated water in normal placentae

\begin{tabular}{|c|c|c|c|c|c|c|}
\hline Substance & $\begin{array}{l}\text { AUC, } \\
\text { dpm }\end{array}$ & $\begin{array}{l}\text { Clearance, } \\
\mathrm{ml} / \mathrm{min}\end{array}$ & $\mathrm{K}_{\mathrm{el}}$ & $\mathrm{T}_{\max }, \mathrm{s}$ & $\begin{array}{l}\text { Absorption } \\
\text { rate, } \text { dpm }\end{array}$ & $\begin{array}{l}\text { Elimination } \\
\text { rate, } \mathrm{dpm}\end{array}$ \\
\hline Tritiated water & $1,143,622 \pm 79,612$ & $2.66 \pm 0.92$ & $5.11 \pm 0.71$ & $95.8 \pm 6.3$ & $969 \pm 98$ & $3,702 \pm 198$ \\
\hline AIB & $56,185 \pm 2,626$ & $45.3 \pm 13.8$ & $3.06 \pm 0.84$ & $124.1 \pm 6.9$ & $87 \pm 29$ & $172 \pm 56$ \\
\hline
\end{tabular}

Values represent means \pm SEM of 5 successful perfusions.

demonstrating free placental permeability for water across the placental membrane in animals $[3,12]$ as well as in humans $[10,13]$. Active transport of AIB across the human placenta has already been firmly established [1416]. Our data on the amino acid transfer are consistent with previous reports on AIB transport in the perfused placental lobule $[1,2,17]$. The AIB TF value of about $0.22 \pm 0.08 \%$ compares favorably with that computed from the data of Contractor and Stannard [1] for a comparable duration. By plotting relative AIB
TR in different perfusate samples as a function of perfusion time, and by determining the correlation between the two variables, we were able to confirm the active nature of AIB transport reported by others. Besides, calculation of the TR constant of the amino acid in the human placenta in vitro under controlled experimental conditions will provide the investigators with a useful tool to investigate amino acid transport function in a wide variety of experimental conditions as well as in pregnancy disease states. 
Expression of the results as percentage of the cumulative efflux in the fetal vein and plotting of data as function of perfusion time have permitted us to delineate the functional characteristics of the human placental membrane. Control studies have shown that the efflux curve pattern as well as the differential TR of the test substances remained unchanged with regard to the injected dose as well as the perfusion flow rates when minor alterations were introduced in the protocol. However, it was observed in the control studies that wide variations in the flow rates brought about appreciable changes in the pattern of efflux, resulting in wide variations in the differential TR. For this reason, for a given study, the flow rates were kept relatively constant in both the fetal as well as the maternal circuits. A similar variation, however, was not evident when the injected dose was doubled and tripled for a given set of flow rates. Computation of kinetic parameters as AUC, clearance, $\mathrm{K}_{\mathrm{el}}, \mathrm{T}_{\max }$, absorption rate and elimination rate, and expression of the results as ratio of the corresponding tritiated water values have also helped us to delineate membrane characteristics with respect to AIB transport behavior under the specified study conditions. The AUC index value of the ami- no acid $(12 \pm 2 \%)$ was not significantly different from the TF index of $13.2 \pm 1.8 \%$. Interestingly, the AUC index as well as the TF index of the amino acid compare well with its absorption rate index expressed in relation to that of the reference marker. It is logical to assume that parameters such as the $\mathrm{TR}_{50}$ index, relative TR and transport kinetic indices are valuable tools permitting assessment of the dynamic transport function of the placental membrane for a given set of study substances and reference markers in varying experimental conditions as well as in various disease states. This parameter can also be usefully employed to assess transport behavior of A type amino acids [18] in varying experimental conditions. Currently, studies are in progress to evaluate possible alterations in the dynamic placental membrane function with regard to major metabolic fuels in various pregnancy-related disease states.

\section{Acknowledgment}

The authors are grateful to Mrs. Nirmala Narayanan and Dr. K.S. Sujatha for their excellent technical help. The work was supported by the Kuwait University Research Grant No. M0026.

\section{References}

1 Contractor SF, Stannard PJ: The use of AIB transport to assess the suitability of a system of human placental perfusion for drug transport studies. Placenta 1983;4:19-29.

2 Schneider H, Proegler M, Sodha R, Dancis J: Asymmetrical transfer of alpha-aminoisobutyric acid (AIB), leucine and lysine across the in vitro perfused human placenta. Placenta 1987;8:141-151.

Placental Transport Kinetics of

$\alpha$-Aminoisobutyric Acid
3 Ventura MA, Gardey C, Thiroux G, Nandakumaran M, Rey E, Richard MO: Effect of propranolol on alphaaminoisobutyric acid and water kinetics in the materno-fetal unit of the rat. Dev Pharmacol Ther 1986; 9:266-281.

4 Copeland AD, Porterfield SP: Effect of streptozotocin induced diabetes in pregnant rats on placental transport and tissue uptake of alpha amino-isobutyric acid. Horm Metab Res 1987;19:57-61.
5 Nandakumaran M, Sugathan TN: Assessment of transport dynamics in the perfused human placental lobule. Med Principles Pract 1992-93; 3:219-222.

6 Nandakumaran M, Makhseed M, Devarajan LV: Transport of water and inulin in insulin-dependent diabetic pregnancies: In vitro study. Diabetes Res 1995;28:9-15. 
7 Panigel M, Pascaud M, Brun JL: Une nouvelle technique de perfusion de l'espace intervilleux dans le placenta humain isolé. Pathol Biol 1967;15:821.

8 Schneider H, Panigel M, Dancis J: Transfer across the perfused human placenta of antipyrine, sodium and leucine. Am J Obstet Gynecol 1972; 114:822-828.

9 Nandakumaran M, Gardey C, Challier JC, Panigel M, Olive G: Transfer of salbutamol in the human placenta in vitro. Dev Pharmacol Ther 1981;3:88-98.

10 Nandakumaran M, Gardey C, Rey E, Challier JC, Panigel M, Olive G: Transfer of ritodrine and norepinephrine in the human placenta in vitro. Dev Pharmacol Ther 1982;4: 71-80.
11 Rey E, Nandakumaran M, Richard MO, Loose JP, D'Athis P, SaintMaurice C, Olive G: Pharmacokinetics of flunitrazepam after single rectal administration in children. Dev Pharmacol Ther 1984;7(suppl 1):206-212.

12 Meschia G, Battaglia FC, Bruns PD: Theoretical and experimental study of transplacental diffusion. J Appl Physiol 1967;22:1171-1178.

13 Nandakumaran M, Challier JC, Rey E, Richard MO, Olive G: In vitro transfer of six benzamides in the human placenta. Dev Pharmacol Ther 1984; 7:60-66.

14 Miller RK, Berndt WO: Characterisation of neutral amino acid accumulation by human term placental slices. Am J Physiol 1975;227: 1236-1242.
15 Young M: Transfer of amino acids; in Chamberlain G, Wilkinson A (eds): Placental Transfer. Toronto, Pitman Medical, 1979, pp 142-158.

16 Wier PJ, Miller RK, Maulik D, Di Sant'Agnese PA: Bidirectional transfer of alpha-aminoisobutyric acid by the perfused human placental lobule. Trophobl Res 1983;1:3754.

17 Krishna BR, King RG, Brennecke SP: Acetylcholine output and materno-fetal alpha-amino-isobutyric acid transfer in the perfused human placental lobule. Reprod Fertil Dev 1991;3:459-465.

18 Enders RH, Judd RM, Donohue TM, Smith TH: Placental aminoacid uptake. III. Transport systems for neutral aminoacids. Am J Physiol 1976;230:706-710. 